\title{
Limitations of rapid parallel processing in the detection of long and short oriented line targets
}

\author{
LAURA M. DOHERTY ${ }^{1 . *}$ and DAVID H. FOSTER ${ }^{2}$ \\ ${ }^{1}$ MacKay Institute of Communication and Neuroscience, Keele University, \\ Staffordshire ST5 $5 B G, U K$ \\ ${ }^{2}$ Visual Sciences Laboratory, Department of Optometry and Neuroscience, University of Manchester \\ Institute of Science and Technology, Manchester M60 IQD, UK
}

Received 6 December 1998; revised 23 April 1999; accepted 26 April 1999

\begin{abstract}
The purpose of this study was to determine whether the detectability of a uniquely oriented line element in a field of uniformly oriented line elements depends on element length. Displays containing various numbers of elements were presented briefly and followed by a mask. The length and orientation of the elements were varied. With longer (1.0-deg) elements, detection performance varied little with the number of elements present. With shorter $(0.25-\mathrm{deg})$ elements, performance worsened as the element number increased, especially when the uniformly oriented elements were oblique. It seems that rapid spatially parallel processes facilitate detection of targets in many-element displays of long elements but not of short elements.
\end{abstract}

Keywords: Visual search; parallel processing; oricntation; line targets; prcattentive vision.

\section{INTRODUCTION}

Lines and edges of different orientations can be easily detected in images lasting only a few hundred milliseconds. The early visual processes underlying this ability have been studied in visual-search and target-detection paradigms in which the task of the observer is to detect a uniquely oriented line element ('target') among uniformly oriented line elements ('nontargets'). In these studies, differing inferences have been made concerning the effectiveness of rapid spatially parallel visual processes in this task (e.g. Treisman and Gormican, 1988; Marendaz et al., 1991; Verghese and Nakayama, 1994). The purpose of the present work was to determine whether the use of different line-element lengths could be an explanatory factor, as element length has previously been found to affect orientation discrimination (Weymouth, 1959; Scobey, 1982; Mäkelä et al., 1993).

\footnotetext{
"E-mail: 1.m.doherty@ cns.keele.ac.uk
} 


\section{I. Identifying the processes involved in search and detection}

The variation of detection performance with the number of elements in the display, the 'set size', has been taken to indicate the effectiveness of parallel processes (e.g. Treisman and Gelade, 1980; Sagi and Juleš, 1987). Performance measures used include reaction times (in visual-search experiments where the search display remains visible until the observer responds) and orientation increment thresholds or percentage of correct responses (in target-detection experiments where serial scanning of the elements is prevented by brief presentation and masking of the targetdetection display). If performance measured in one of these ways remains stable as the set size is increased, then it can be inferred that it is determined by rapid spatially parallel processes. If performance worsens as the set size is increased, then it can be inferred that these processes are inadequate and detection is mediated by slower parallel processes or by serial processes. In studies of visual search, an increase in reaction time with increasing set size has often been taken as evidence of serial processing of information from different locations (e.g. Treisman and Gelade, 1980; Marendaz et al., 1991), although examination of search performance in a wide range of tasks has yielded no evidence of an abrupt transition from parallel to serial processing (Wolfe, 1998). It has been argued that serial processing and limited-capacity parallel processing could produce similar increases in response time with set size (Townsend, 1971), and that these increases are due to the time taken for integration of information from multiple stimuli at the decision stage of processing, rather than the use of qualitatively different (parallel or serial) perceptual strategies (Palmer, 1994). Distinguishing between serial and slow parallel processes as causes of increased response time is beyond the scope of the present study, as the experimental paradigm-target-detection with brief, masked displays - was designed to test the use of rapid parallel processes. In such a paradigm, an increase in increment threshold or a decrease in the percentage of correct responses with increasing set size is taken simply to imply that the information extracted rapidly and in parallel from many locations is less precise than that extracted rapidly and in parallel from fewer locations. (It should be noted that with brief, masked displays the increment thresholds are likely to be greater than those obtained in paradigms in which the observer views the image for longer durations. Attentional effects in orientation discrimination, such as those reported by Lee $e t$ al. (1997), are also unlikely to be found with brief-presentation paradigms, as observers have insufficient time to redistribute their attention during the presentation.)

\subsection{Parallel processing in oriented-line-target detection}

Previous experiments have yielded conflicting evidence concerning the ability of observers to detect oriented line targets using only rapid parallel processes. In some studies of visual search for a vertical target among tilted nontargets (oriented at 18 deg to the vertical), reaction time has been found to increase with 
increasing set size, a result which would be expected if rapid parallel processes were inadequate (Treisman and Gormican, 1988; Marendaz et al., 1991). In visual search for a tilted target among vertical nontargets, rapid parallel processes were apparently adequate (Treisman and Gormican, 1988; Marendaz et al., 1991). Thus performance was found to be anisotropic with respect to nontarget orientation, to the extent that a change in nontarget orientation could produce a change from rapid parallel processing to slower (serial or slow parallel) processing. ${ }^{1}$ In other studies of visual search (Javadnia and Ruddock, 1988; Found and Müller, 1997), performance was found to be anisotropic in that reaction times were greater with oblique nontargets ( $45 \mathrm{deg}$ to the vertical) than with vertical nontargets; but, unlike the studies of Treisman and Gormican (1988) and Marendaz et al. (1991), these studies suggested that processing could be parallel with either oblique or vertical nontargets when the difference between nontarget and target orientations was $10 \mathrm{deg}$ or more.

In some studies of target detection with brief, masked displays and vertical nontargets, performance measured by orientation increment threshold (Verghese and Nakayama, 1994; Palmer, 1994, who used ellipses rather than line elements) and percentage of correct responses (Sagi and Julesz, 1987) was found to worsen with increasing set size. But, in another study with brief, masked displays and vertical, horizontal, or tilted nontargets, performance was found not to worsen with increasing set size (Doherty and Foster, 1995). In that study, performance measured by orientation increment threshold was poorer with tilted nontargets than with vertical or horizontal nontargets, but the stability of performance with respect to changes in set size was consistent with rapid parallel processing at all nontarget orientations.

In a study that suggested a worsening of performance with increasing set size even when the orientation increment was large (Verghese and Nakayama, 1994), line elements subtended 0.25 deg and observers knew the set of locations at which the target could appear. In the studies in which no such worsening was found (Javadnia and Ruddock, 1988; Doherty and Foster, 1995), line elements were longer (subtending 0.45 and $1.0 \mathrm{deg}$, respectively) and observers had no prior knowledge of target location. Thus, some differences in set-size effects could have arisen because of differences in line-element length or knowledge of target position. Individual differences in orientation-processing characteristics might also have been important. In the present experiment, line-target-detection performance was measured with different line-element lengths; the observers, unlike those in the study of Verghese and Nakayama (1994), were given no information about element orientations or positions. It was found that reducing line-element length reduced the effectiveness of rapid parallel processing and that the strength of the orientational anisotropy in detection performance varied markedly between observers. 


\section{METHODS}

\subsection{Stimuli and apparatus}

Line elements had one of two lengths, with only one length being used in each experimental session. In terms of angular subtense, long elements had length $1.0 \mathrm{deg}$ and width $0.1 \mathrm{deg}$, and were located randomly within a circular field of diameter $20 \mathrm{deg}$. The target, if present, appeared within an annulus of inner radius $3 \mathrm{deg}$ and outer radius $8 \mathrm{deg}$. Displays containing 5 or fewer long elements had a minimum element spacing of $5 \mathrm{deg}$ and displays containing 10 or 20 long elements had a minimum element spacing of $2 \mathrm{deg}$. Displays with long elements were viewed from a distance of $0.5 \mathrm{~m}$. Short elements had length $0.25 \mathrm{deg}$ and width $0.05 \mathrm{deg}$ and were located randomly within a circular field of diameter $10 \mathrm{deg}$. The target, if present, appeared within an annulus of inner radius $1.5 \mathrm{deg}$ and outer radius 4 deg. (The length of the short elements was within 0.01 deg of that used by Verghese and Nakayama (1994) and the element densities and target eccentricities were also similar to those in that study.) Displays containing 5 or fewer short elements had a minimum element spacing of $2.5 \mathrm{deg}$ and displays containing 10 or 20 short elements had a minimum elements spacing of $1.0 \mathrm{deg}$. Displays with short elements were viewed from a distance of $1.15 \mathrm{~m}$. In all conditions, the probability of a target being present was 0.5 . A masking display was generated by placing a cluster of 4 randomly oriented elements at the location of each element in the target-detection display. The lengths of these randomly oriented elements were the same as in the target-detection display.

Displays were produced on a cathode-ray tube (Hewlett-Packard, Type 1321A with white P4 sulfide phosphor or Type 1317A with green P31 phosphor, each with $90-10 \%$ decay times of less than $1 \mathrm{~ms}$ ) controlled by a true-line vectorgraphics generator (Sigma Electronic Systems, QVEC 2150) and additional digitalto-analogue converters, in turn controlled by a laboratory computer. (The choice of phosphor colour, green or white, had been previously found not to affect detection performance; Westland and Foster, unpublished data.) Each display was refreshed at intervals of $20 \mathrm{~ms}$. (This temporal structure was not visually detectable.)

This system produced very-high-resolution line-element displays in which individual line elements were defined with endpoint (linear) resolutions of 1 part in 1024 over an imaginary square patch of side $1.25 \mathrm{~cm}$. Each patch was located with a precision of 1 part in 4096 over the $17-$ in $(43-\mathrm{cm})$ CRT screen. The accuracy of local positioning was verified with a travelling microphotometer. Orientation accuracy was differentially better than $0.2 \mathrm{deg}$ and absolutely better than $0.5 \mathrm{deg}$. The intensity of the elements did not vary with their orientations (see Foster and Westland (1998) for further details). Before each experimental session the CRT was allowed to warm up for at least $20 \mathrm{~min}$ and its spatial calibration was then verfied by aligning a test image against a transparent template that was placed over the screen.

Observers viewed the CRT through a view-tunnel providing a uniform background field with luminance about $35 \mathrm{~cd} \mathrm{~m}^{-2}$; at the beginning of each experimental 
session, the luminance of the line elements was set for each observer to about $1 \log$ unit above increment threshold on this field.

The display configurations were designed to allow a comparison of the experiments of Verghese and Nakayama (1994) and Doherty and Foster (1995). Shortelement and long-element displays therefore differed not only in terms of element length but in terms of target eccentricity, element width and aspect ratio, and spatial density. The effects of these factors are considered later.

\subsection{Design}

For each line-element length, there were five possible set sizes $(2,3,5,10$, and 20 elements) and two possible nontarget orientations (oblique, i.e. rotated $45 \mathrm{deg}$ clockwise from the vertical, and vertical). The nontarget orientation was randomly selected in each trial. The orientation increment, that is, the angle added to the nontarget orientation to give the target orientation, varied between $2.5 \mathrm{deg}$ and $40 \mathrm{deg}$ anticlockwise according to the adaptive procedure PEST (Taylor and Creelman, 1967; modified by Hall, 1981). Separate interleaved PEST procedures were used for the two nontarget orientations. These procedures were initially set to converge to $66 \%$ correct. In order to ensure sufficient sampling to fit a psychometric function, this convergence point was adjusted in some sessions if necessary. Sessions with long elements and with short elements were ordered randomly. Each session lasted about $35 \mathrm{~min}$ and comprised 600 trials, grouped into blocks of 120 trials each. In each block, the set size remained constant. Each session comprised one block of each set size, and blocks were randomly ordered.

\subsection{Procedure}

At the beginning of each trial, a fixation cross appeared at the centre of the CRT screen. The observer pressed a button on a pushbutton switch-box held in the nondominant hand. After a 20 -ms interval, the target-detection display was presented for $40 \mathrm{~ms}$. Then an interval followed during which the screen was blank. The duration of the blank interval remained constant for each observer throughout the experiment, and was set at 60,120 , or $180 \mathrm{~ms}$, whichever was the shortest duration yielding about $66 \%$ correct detection during practice sessions with 20 long elements. After this interval, the masking display was presented for $500 \mathrm{~ms}$. The observer indicated whether a target was present by pressing one of two buttons on a pushbutton switch-box held in the dominant hand.

\subsection{Observers}

There were four observers, aged between 20 and $28 \mathrm{yr}$. All had normal or correctedto-normal visual acuity (Snellen acuity 6/6 or better) and optometrically verified residual astigmatism of not more than $0.25 \mathrm{D}$. Three (one was co-author LD) had participated in similar psychophysical experiments; the fourth had not and 
completed four practice sessions on the target-detection task before participating in the study. Observers were given no feedback on their performance in the task.

\subsection{Analysis}

Each observer's response performance was summarized by the discrimination index $d^{\prime}$ from signal-detection theory (Green and Swets, 1966). For each nontarget orientation, the value of $d^{\prime}$ and its variance were calculated for each increment between nontarget and target orientations selected by the PEST procedure. For each condition defined by element length and set size, there were about 240 trials per nontarget orientation. The resulting data were adequately fitted with linear functions by a weighted least-squares procedure (over all conditions and observers, $\chi_{587}^{2}=357, p>0.5$ ). For each nontarget orientation, the threshold was taken to be the orientation increment for which the fitted functions gave a value of $d^{\prime}=0.5$, which corresponds to $66 \%$ correct for an unbiased observer in a Yes-No task. Deriving thresholds from the psychometric function in this way is more efficient than using the threshold estimates from PEST directly (Hall, 1981). The standard errors of these thresholds were estimated using a bootstrap procedure (Foster and Bischof, 1997), which takes account of the variance associated with each $d^{\prime}$ value.

\section{RESULTS AND COMMENT}

\subsection{Effects of line-element length and nontarget orientation}

Figure 1 shows the variation of orientation increment threshold with set size for each experimental condition (columns) and observer (rows). The straight lines are least-squares linear fits. These functions did not always provide good descriptions of the data but do indicate whether, overall, there was an increase in threshold with increasing set size. With long elements (first two columns, Fig. 1), the gradients of the linear functions were generally close to zero. The only gradients differing significantly from zero were for BF with vertical nontargets $(0.16$ deg per element for an average threshold of $5.0 \mathrm{deg}, p<0.05)$ and NL with oblique nontargets (0.31 deg per element for an average threshold of $9.1 \mathrm{deg}, p<0.0001)$. With short elements (third and fourth columns, Fig. 1), all of the gradients were positive and differed significantly from zero $(p<0.05$ for observer LD with vertical nontargets and for observers BF and DP with oblique nontargets; $p<0.001$ for observers $\mathrm{BF}$ and NL with vertical nontargets; $p<0.0001$ for all other combinations of observer and nontarget orientation). The gradients obtained with short elements and oblique nontargets were either similar to those obtained with short elements and vertical nontargets (observers BF and DP) or greater (observers NL and LD). When the set size was 2, thresholds with short elements were usually greater than those with long elements, although the difference in threshold was not always significant. With larger set sizes, thresholds with short elements were greater than 


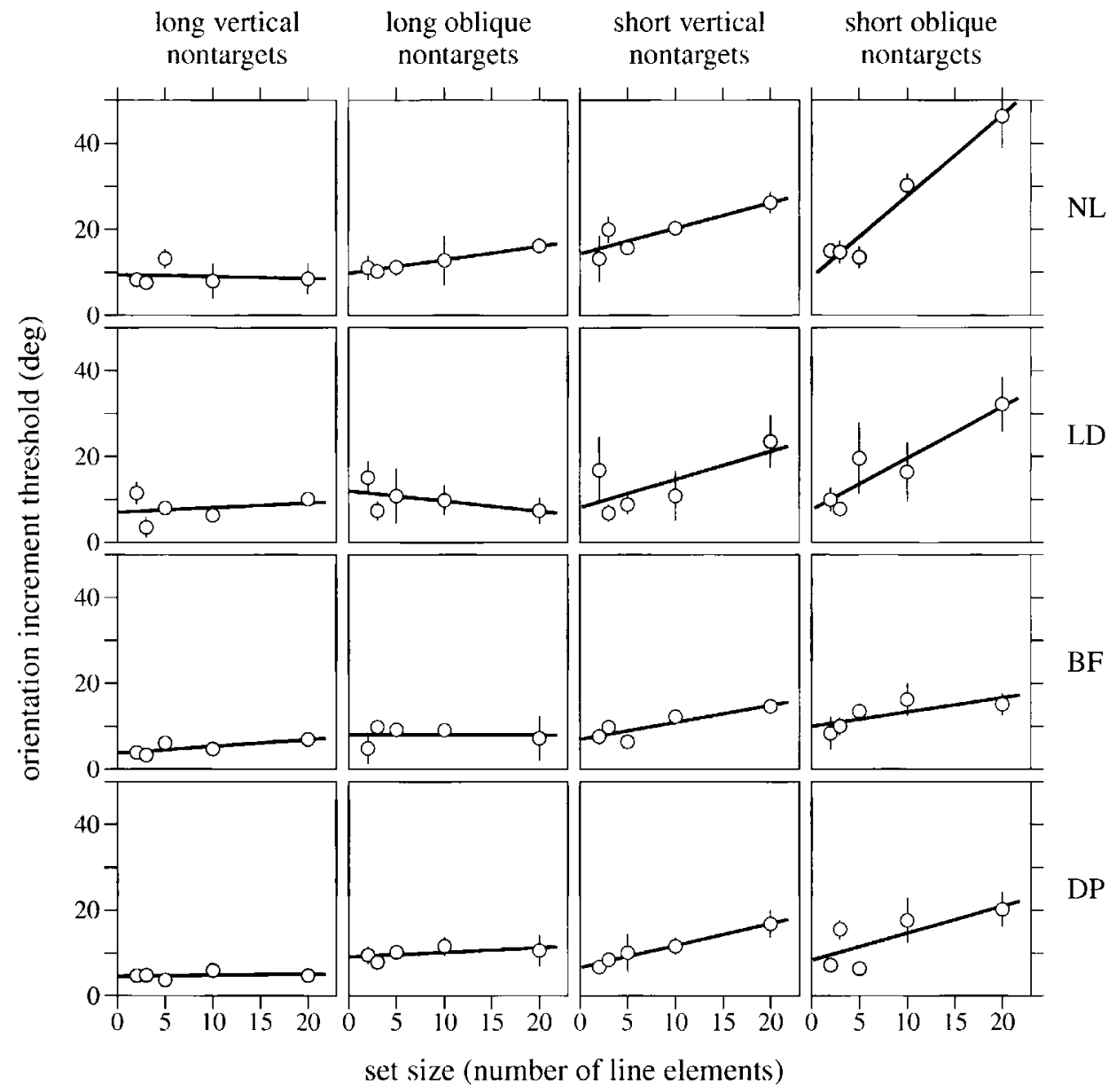

Figure 1. Variation of orientation increment threshold with set size. The first column shows data for displays with long vertical nontargets, the second for displays with long oblique nontargets, the third for short vertical nontargets, and the fourth for short oblique nontargets. The top, second, third, and bottom rows show data for observers NL, LD, BF, and DP, respectively. Solid lines show least-squares linear fits. Error bars show $\pm \mathrm{I}$ bootstrap estimates of the standard error.

those with long elements with only one exception (observer DP, 5 elements, oblique nontargets). More of the differences in thresholds with short and long elements reached significance with large than with small set sizes.

\subsection{Learning effects}

As there have been several reports of learning in oriented-line-target detection (e.g. Karni and Sagi, 1991; Schoups and Orban, 1996; Ahissar and Hochstein, 1997), the present data were tested for practice effects. For each condition defined by observer, set size, and nontarget orientation, the orientation increment threshold obtained from data from the first two blocks was compared with that obtained from 
data from the last two blocks. Of the 80 comparisons made, 5 revealed significantly different thresholds $(p<0.05)$. In 4 of these 5 comparisons the threshold for the first two blocks was higher than that for the second two blocks. So, although there was evidence of learning in a very few conditions, it seems unlikely that learning had an important influence on the general level of detection performance during the course of the experiment. This failure to find a strong effect of learning is consistent with the findings of Ahissar and Hochstein (1997), who found that learning in tasks with small orientation increments ( 20 deg or less, as in most trials in the present study) tended to be position-specific: such learning would not have been effective in the present study as the target was placed randomly in one of very many locations.

\subsection{Effect of target eccentricity}

Rapid texture-segregation has been found to be poorer with stimuli at the fovea than with stimuli in the periphery (Kehrer, 1987): if a similar effect holds for target detection, then the proximity of short-element targets to the fixation point might have led to an increase in threshold due to eccentricity rather than to element length. For each condition defined by observer, set size, and nontarget orientation, the orientation increment threshold obtained from trials with targets close to fixation (central targets: between 3 deg and 5.5 deg from fixation for long-element displays and between 1.5 and $2.8 \mathrm{deg}$ from fixation for short-element displays) was compared with the threshold obtained from trials with targets further from fixation (peripheral targets: between $5.5 \mathrm{deg}$ and $8.0 \mathrm{deg}$ from fixation for long-element displays and between $2.8 \mathrm{deg}$ and $4.0 \mathrm{deg}$ from fixation for short-element displays). With longelement displays, 4 of the 40 comparisons made revealed significant differences in threshold $(p<0.05)$. In all 4 of these comparisons the threshold for the peripheral targets was greater than that for the central targets. With short-element displays, 6 of the 40 comparisons made revealed significant differences in threshold $(p<0.05)$. In all 6 of these comparisons the threshold for the peripheral targets was greater than that for the central targets. So, whenever there was a significant effect of target eccentricity, detection was better with targets close to fixation than with those further from fixation (similar eccentricity effects have been found for other visual tasks; e.g. Mäkelä et al., 1993; Carrasco and Frieder, 1997). Poorer detection with short-element than with long-element displays seems not to be attributable to the difference in the retinal eccentricity of the targets.

\subsection{Effect of aspect ratio}

As mentioned previously, short-element and long-element displays differed in terms of element aspect ratio. Long elements had an aspect ratio of $10: 1$ and short elements had an aspect ratio of $5: 1$. To test whether there was an effect of aspect ratio, one observer (LD) performed a control experiment in which the element length was $0.25 \mathrm{deg}$ and the aspect ratio was either $5: 1$ or $10: 1$. Displays contained 10 elements, the blank inter-stimulus interval lasted $60 \mathrm{~ms}$, and different aspect 
ratios were produced by adjusting the viewing distance. Orientation increment thresholds, obtained in the same way as in the main experiment, did not differ significantly, and showed no consistent effect of aspect ratio. (With vertical nontargets, the thresholds were $14.9 \pm 3.5 \mathrm{deg}$ with an aspect ratio of $5: 1$ and 13.1 $\pm 6.4 \mathrm{deg}$ with an aspect ratio of $10: 1$. With oblique nontargets, the thresholds were $15.2 \pm 2.7 \mathrm{deg}$ with an aspect ratio of $5: 1$ and $16.5 \pm 1.7 \mathrm{deg}$ with an aspect ratio of $10: 1$.) Differences in aspect ratio seem unlikely to have been important in the present study.

\subsection{Spatial element density}

Long-element and short-element displays also differed in spatial element density. With set sizes greater than 20, detection has been found (Sagi and Julesz, 1987) to be better with a minimum inter-element spacing equal to twice the element length (as in the long-element displays with set sizes 10 and 20 in the present study) than with inter-element spacings greater than twice the element length (as in the other displays in the present study). With smaller set sizes, the variation in detection with set size seemed unaffected by density. On the basis of that result, no important density effects would be expected in the present study, as all displays contained 20 elements or fewer. (The effects of element density with spatially bandlimited stimuli have been examined (Sagi, 1990), but it is unclear whether those effects can be used to predict performance with the spatially broad-band stimuli used in the present experiment.) To determine whether the density difference between shortelement and long-element displays affected detection, one observer (LD) performed a control experiment in which the element length was $0.25 \mathrm{deg}$ and the ratio of the minimum element element spacing to the element length was either $2: 1$ or $4: 1$. Displays contained 20 elements (this large set-size was used because the density effects previously reported were found with large set-sizes), and the blank interstimulus interval lasted $60 \mathrm{~ms}$. Orientation increment thresholds, obtained in the same way as in the main experiment, did not differ significantly, and showed no consistent effect of density. (With vertical nontargets, the thresholds were $13.2 \pm$ $3.2 \mathrm{deg}$ with a spacing-to-length ratio of $2: 1$ and $15.9 \pm 3.4 \mathrm{deg}$ with a spacing-tolength ratio of $4: 1$. With oblique nontargets, the thresholds were $35.8 \pm 16.2 \mathrm{deg}$ with a spacing-to-length ratio of $2: 1$ and $28.6 \pm 6.9 \mathrm{deg}$ with a spacing-to-length ratio of $4: 1$.) Differences in density seem unlikely to have been important in the present study.

\section{DISCUSSION}

\subsection{Effect of line-element length}

With long elements, orientation increment thresholds were generally independent of set size, but with short elements, orientation increment thresholds generally 
increased with increasing set size. This effect would be expected if rapid parallel processes extracted sufficient information for target detection with many-element displays of long elements but not of short elements. One possible explanation for the failure of these processes with short elements is that the orientations of short elements are represented less precisely by orientation-selective filter mechanisms than the orientations of long elements.

The finding that reducing element length reduces the effectiveness of rapid parallel processing might explain why the study by Verghese and Nakayama (1994), in which elements were short $(0.25 \mathrm{deg})$, showed a worsening in performance with increasing set size whereas those studies in which elements were longer $(0.45$ or $1.0 \mathrm{deg}$ ) showed no such effect (Javadnia and Ruddock, 1988; Doherty and Foster, 1995). This result may have implications for some models of early line-orientation coding (Bergen and Julesz, 1983; Malik and Perona, 1990; Westland and Foster, 1995); for instance, the constrained spatial resolution of rapid parallel processes should be considered when applying models of early orientation-processing at many scales. The analysis of the present data was not, however, predicated on a specific model of line-target detection: such a model would need to include estimates of the number and spatiotemporal characteristics of orientation-sensitive mechanisms, the variation in sensitivity with retinal position, the nature of the integration of signals across mechanisms and locations, and possibly some parameters to describe overall processing capacity and observer differences. Simpler models, although tractable, do not always predict search performance well (Rosenholtz, 1997). There are many models of rapid texture-processing (e.g. Gurnsey and Browse, 1989; Malik and Perona, 1990; Rubenstein and Sagi, 1990) and target-detection (Foster and Ward, 1991; Westland and Foster, 1995) that successfully account for specific experimental data or provide information about underlying mechanisms, but none is sufficiently comprehensive to straightforwardly predict performance in the present study. The dependence of target-detection performance on element length accounts for some of the apparent differences in processing (rapid and parallel, or slower), but not all. In one target-detection experiment (Sagi and Julesz, 1987), performance was found to worsen with increasing set size even when elements were long ( $1.0 \mathrm{deg}$ ) and orientation increments were large ( $45 \mathrm{deg}$ or more). It is unclear whether this result was a consequence of a particular combination of element orientations and performance measure, or whether observers differ widely in their ability to extract orientation information using only rapid parallel processes.

\subsection{Effect of nontarget orientation}

With long elements the expected orientational anisotropies were found: thresholds for detecting targets among oblique nontargets were almost always higher than those for targets among vertical nontargets. With short elements there were also orientational anisotropies: for most observers in most conditions, thresholds were greater with oblique than with vertical nontargets. The strength of the anisotropy (indicated in part by the effectiveness of parallel processes when nontargets were 
oblique) varied between observers. For observer NL with long (1.0-deg) line elements, rapid parallel processes seemed adequate with vertical nontargets, and slower processes seemed necessary when nontargets were oblique. For observers DP and LD, rapid parallel processes were adequate when elements were long, both when nontargets were vertical and when nontargets were oblique.

\section{CONCLUSION}

It is clear from studies of search and detection with targets defined by various attributes such as orientation, colour, and motion that the nature of the detection process depends on the attribute defining the target (Nothdurft, 1993; Verghese and Nakayama, 1994). The present work has shown that the nature of the detection process depends also on attributes shared by all elements in the display, and that it is essential to consider the effects of specific stimulus properties when developing descriptions of this process (cf. Palmer, 1994). It seems that rapid spatially parallel processes facilitate detection of targets in many-element displays of long elements but not of short elements. It also seems that the effectiveness of these processes may vary from observer to observer.

\section{NOTE}

1. A similar performance asymmetry has been found with horizontal and tilted elements: search performance (Marendaz et al., 1991) and detection performance (Foster and Ward, 1991; Foster and Westland, 1998) are better with horizontal than with tilted nontargets. The asymmetry in detection performance with vertical and tilted elements has been found with sinewave-grating elements as well as with line elements (Poirier and Gurnsey, 1988).

\section{Acknowledgements}

We thank K. J. Linnell for comments on the manuscript. Much of the work reported here was carried out while the authors were in the Department of Vision Sciences, Aston University, Birmingham, UK. Support was provided by the Engineering and Physical Sciences Research Council (UK) and the Wellcome Trust (grant no. 039958).

\section{REFERENCES}

Ahissar, M. and Hochstein, S. (1997). Task difficulty and the specificity of perceptual learning, Nature 387, 401-406.

Bergen, J. R. and Julesz, B. (1983). Parallel versus serial processing in rapid pattern-discrimination, Nature 303, 696-698. 
Carrasco, M. and Frieder, K. S. (1997). Cortical magnification neutralizes the eccentricity effect in visual search, Vision Res. 37, 63-82.

Doherty, L. M. and Foster, D. H. (1995). Detection of oriented line targets in very sparse displays, Perception (Supplement) 24, 132.

Foster, D. H. and Bischof, W. F. (1997). Bootstrap estimates of the statistical accuracy of thresholds obtained from psychometric functions, Spatial Vision 11, 135-139.

Foster, D. H. and Ward, P. A. (1991). Asymmetries in oriented-line detection indicate two orthogonal filters in early vision, Proc. Roy. Soc. Lond. B 243, 75-81.

Foster, D. H. and Westland, S. (1998). Multiple groups of orientation-selective visual mechanisms underlying rapid orientated-line detection, Proc. Roy. Soc. Lond. B 265, 1605-1613.

Found, A. and Müller, H. J. (1997). Local and global orientation in visual search, Percept. Psychophysics 59, 941-963.

Green, D. M. and Swets, J. A. (1966). Signal Detection Theory and Psychophysics. Wiley, New York.

Gurnsey, R. and Browse, R. A. (1989). Asymmetries in visual texture discrimination, Spatial Vision 4, 31-44.

Hall, J. L. (1981). Hybrid adaptive procedure for estimation of psychometric functions, J. Acoust. Soc. Amer: 69, 1763-1769.

Javadnia, A. and Ruddock, K. H. (1988). The limits of parallel processing in the visual discrimination of orientation and magnification, Spatial Vision 3, 97-114.

Karni, A. and Sagi, D. (1991). Where practice makes perfect in texture discrimination: Evidence for primary visual cortex plasticity, Proc. Natl. Acad. Sci. USA 88, 4966-4970.

Kehrer, L. (1987). Perceptual segregation and retinal position, Spatial Vision 2, 247-261.

Lee, D. K., Koch, C. and Braun, J. (1997). Spatial vision thresholds in the near absence of attention, Vision Res. 37, 2409-2418.

Mäkelä, P., Whitaker, D. and Rovamo, J. (1993). Modelling of orientation discrimination across the visual field, Vision Res. 33, 723-730.

Malik, J. and Perona, P. (1990). Preattentive texture discrimination with early vision mechanisms, J. Opt. Soc. Amer. A 7, $923-932$.

Marcndaz, C., Stivalet, P. and Genon, D. (1991). Coding of orientation in early vision: Search asymmetry revisited, European Bull. Cognitive Psychol. 11, 427-440.

Nothdurft, H. C. (1993). The role of features in preattentive vision: Comparison of orientation, motion and color cues, Vision Res. 33, 1937-1958.

Palmer, J. (1994). Set-size effects in visual search: the effect of attention is independent of the stimulus for simple tasks, Vision Res. 34, $1703-1721$.

Poirier, F. J. A. M. and Gurnsey, R. (1998). The effects of eccentricity and spatial frequency on the orientation discrimination asymmetry, Spatial Vision 11,349-366.

Rosenholtz, R. (1997). Basic signal detection theory model for visual search does not predict performance with heterogeneous distractors, Invest. Ophthalmol. Visual Sci. 38, S687.

Rubenstein, B. S. and Sagi, D. (1990). Spatial variability as a limiting factor in texture-discrimination tasks: implications for performance asymmetries, J. Opt. Soc. Amer. A 7, 1632-1643.

Sagi, D. (1990). Detection of an orientation singularity in Gabor textures: effect of signal density and spatial-frequency, Vision Res. 30, 1377-1388.

Sagi, D. and Julesz, B. (1987). Short-range limitation on detection of feature differences, Spatial Vision 2, 39-49.

Schoups, A. A. and Orban, G. A. (1996). Interocular transfer in perceptual learning of a pop-out discrimination task, Proc. Natl. Acad. Sci. USA 93, 7358-7362.

Scobey, R. P. (1982). Human visual orientation discrimination, J. Neurophysiol. 48, 18-26.

Taylor, M. M. and Creelman, C. D. (1967). PEST: Efficient estimates on probability functions, J. Acoust. Soc. Amer. 41, 782-787.

Townsend, J. T. (1971). A note on the identifiability of parallel and serial processes, Percept. Psychophysics 10, 161-163. 
Treisman, A. M. and Gelade, G. (1980). A feature-integration theory of attention, Cognitive Psychol. 12, 97-136.

Treisman, A. and Gormican, S. (1988). Feature analysis in early vision: Evidence from search asymmetries, Psychol. Rev $\mathbf{9 5}, 15-48$.

Verghese, P. and Nakayama, K. (1994). Stimulus discriminability in visual search, Vision Res. 34, 2453-2467.

Westland, S. and Foster, D. H. (1995). Optimized model of oriented-line-target detection using vertical and horizontal filters, J. Opt. Soc. Amer. A 12, 1617-1622.

Weymouth, F. W. (1959). Stimulus orientation and threshold: an optical analysis, Am. J. Ophthalmol. $48,6-10$.

Wolfe, J. M. (1998). What can 1 million trials tell us about visual search? Psychol. Sci. 9, 33-39. 